\title{
The cause of axial rotation of the scoliotic spine
}

\author{
L G Lemmers MSc \\ M M Sanders MSc \\ $\mathrm{J}$ C Cool MSc \\ H J Grootenboer MSc PhD
}

Department of Mechanical Engineering, University of Twente, Enschede, The Netherlands

\begin{abstract}
Summary
To explain the cause of axial rotation in a scoliotic vertebral column, the influence of the gravitation force on a spine with a C-scoliosis has been investigated by means of a mechanical model. In this model the gravitation force takes hold of the three-dimensionally curved vertebral column eccentrically. From these reflections it appears that the axial rotation in the scoliotic spine can be explained by the moment distribution caused by this eccentrical gravitation force. The moment distribution, necessary for correction of the spine, is supposed to be opposite to the moments caused by the gravitation force. The moment distribution caused by the Harrington and the Luque spinal correction systems are compared to the calculated optimum correction moments. It appears that the moment distribution for the Harrington and Luque methods, necessary for the correction of the lateral deviation, are almost the same as the calculated correction moments. But the axial rotation appears to be increasing instead of decreasing in both correction systems.
\end{abstract}

\section{Relevance}

The mechanical analysis as described in this article will be used to design a new system to correct the lateral deviation and the axial rotation of the scoliotic spine.

Key words: Moment diagram, scoliosis, axial rotation, spine, motion segment, axis of axial rotation

$\begin{array}{|llll|}\text { Nomenclature } & & & \\ & & \text { Superscript }^{\mathrm{h}} & \text { Harrington } \\ X, Y, Z & \text { Global coordinates } & \text { Superscript }^{\mathrm{l}} & \text { Luque } \\ x, y, z & \text { Local coordinates } & \text { Force } \\ U X, U Y, U Z & \text { Distances in the global coordinate } & F & \text { Moment } \\ & \text { system } & M & \text { Length of the vertebral column, } \mathrm{L}_{5}-\mathrm{T}_{1} \\ U x, U y, U z & \text { Distances in the local coordinate } & L & \text { Amplitude of the scoliotic curve } \\ & \text { system } & A & \text { Distance to the outer fixation points } \\ \Phi_{x}, \Phi y, \Phi z & \text { Rotations in the local coordinate } & B & \text { (Luque) } \\ & \text { system } & \epsilon & \text { Step function } \\ \text { Superscript } & \text { Gravitation } & & \end{array}$

Received: 13 March 1990

Accepted: 27 October 1990

Correspondence and reprint requests to: Mr Lando Lemmers, University of Twente, Department of Mechanical Engineering (WB/BW), PO Box 217 Enschede, The Netherlands

(C) 1991 Butterworth-Heinemann Ltd

$0268-0033 / 91 / 030179-06$

\section{Introduction}

Scoliosis is a pathological lateral deviaton of the vertebral column coupled with an axial rotation of the vertebrae. Due to this axial rotation the spinous processes rotate towards the concavity of the scoliotic curve. So far, no satisfactory explanation of the axial 


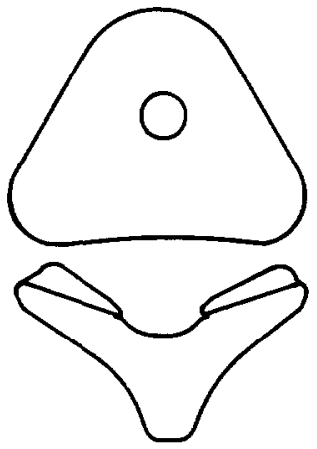

a

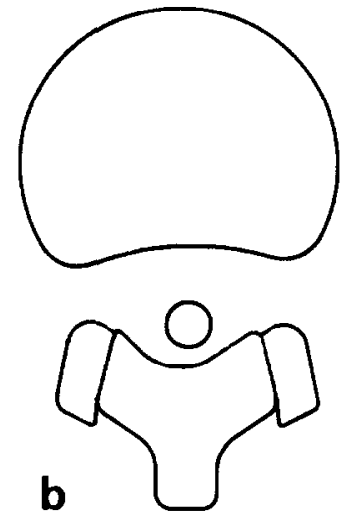

Figure 1. A transverse cross section of two motion segments at the level of the facet joints, showing the axial rotation centres, $O$. a, the rotation centre of a thoracic motion segment; $\mathbf{b}$, the rotation centre of $a$ lumbar motion segment.

rotation of the scoliotic vertebrae has been presented in literature. To assess the cause of the axial rotation is important for the design of a new operative correction system. Therefore, there has been a search for a model to explain and predict the axial rotation in a scoliotic spine.

It is also interesting to consider the influence of the existing correction systems on axial rotation. Therefore the correction for two commonly applied systems. the Harrington and the Luque systems, have been investigated.

\section{Method}

In order to explain the axial rotation, the effect of the gravitation force on the C-scoliotic spine has been modelled and calculated. In this model the position of the axes of axial rotation are important.

\section{Position of the axes of axial rotation}

The axis of axial rotation of a motion segment is the position of a longitudinal axis around which a vertebra rotates with respect to the upper or lower vertebra, when loaded by a moment of torsion.

The location of this axis of rotation is not the same
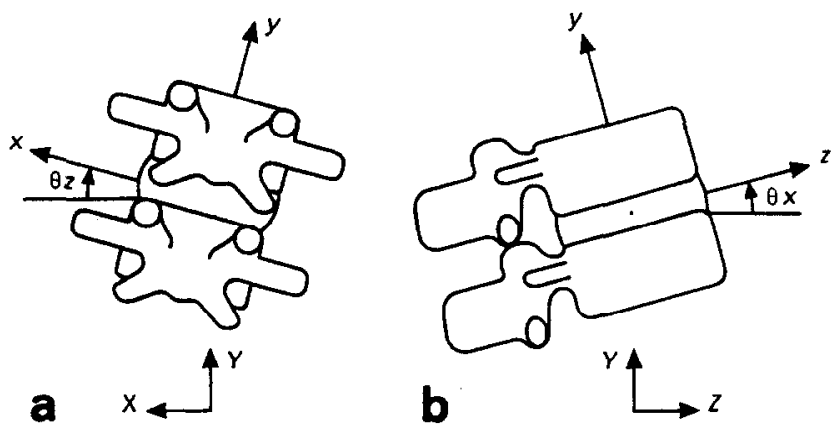

Figure 2. Motion segment with a global and a local coordinate system. for every level of the spine. In the thoracic region the axes of rotation go through the vertebral body whereas the axes in the lumbar region go through the arch. The reason for this shift of the axis of axial rotation is the change of the inclination of the facet joints between $T_{11}$ and $T_{12}{ }^{1-3}$; the axes of axial rotation are mainly determined by the facet joints ${ }^{4.5}$ (Figure 1).

\section{Influence of the gravitation force on the scoliotic spine}

At first a global and a local coordinate system are defined (Figure 2). The global coordinate system is attached to the earth with the origin on the line of action of the gravitation force. The origin of the local coordinate system is positioned in the centre of the intervertebral disc of the motion segment. A motion segment consists of two vertebrae with one intervertebral disc between. The $x$-axis points in the lateral direction, the $y$-axis points in the cranial direction and the $z$-axis in the ventral direction. The Euler angles $\Phi_{z}$ and $\boldsymbol{\Phi}_{x}$ represent respectively the lateral flexion and the sagittal flexion/extension angle of the motion segment. For the transformation from the global to the local coordinate system the following rotation matrix can be derived:

$x=R \times X$

$R=\left[\begin{array}{ccc}\cos \Phi z & \cos \Phi x \sin \Phi z & -\sin \Phi x \sin \phi_{z} \\ -\sin \Phi z & \cos \Phi x \cos \phi z & -\sin \Phi x \cos \Phi z \\ 0 & \sin \Phi x & \cos \phi_{x}\end{array}\right]$

Next the position of the motion segment, with respect to the line of action of the gravitation force has to be considered (see Figure 3 ). The gravitation force in the global coordinate system, the force in the local coordinate system and the resulting moments in the centre of the motion segment are shown in Figure $3 a, b$ and $c$, respectively. With the help of equations (1) and (2) the following local moments can be derived:

$$
\begin{aligned}
& M y(y)=\left(F x^{\mathbb{B}}(y) \times U z(y)\right)-\left(F z^{\mathbb{B}}(y) \times U x(y)\right. \\
& =\left(-F^{\mathrm{s}}(Y) \cos \Phi_{x}(y) \sin \Phi_{z}(y) \times \frac{U Z(Y)}{\cos \Phi_{x(y)}}\right) \\
& -\left(-F^{\mathrm{q}}(Y) \sin \Phi_{x}(y) \times \frac{U X(Y)}{\cos \Phi z(y)}\right) \\
& =\left(-F^{\mathrm{g}}(Y) \sin \Phi z(y) \times U Z(Y)\right. \\
& +\left(F^{\mathbf{g}}(Y) \sin \Phi_{X}(y) \times \frac{U X(Y)}{\cos \Phi_{z(y)}}\right) \\
& M z(y)=F y^{\mathbf{g}}(y) \times U \times(y) \\
& =-F^{\mathbf{B}}(Y) \cos \Phi x(y) \cos \Phi z(y) \times\left(\frac{U X(Y)}{\cos \Phi z(y)}\right) \\
& =-F^{\mathrm{s}}(Y) \cos \Phi_{X}(y) \times U X(Y)
\end{aligned}
$$




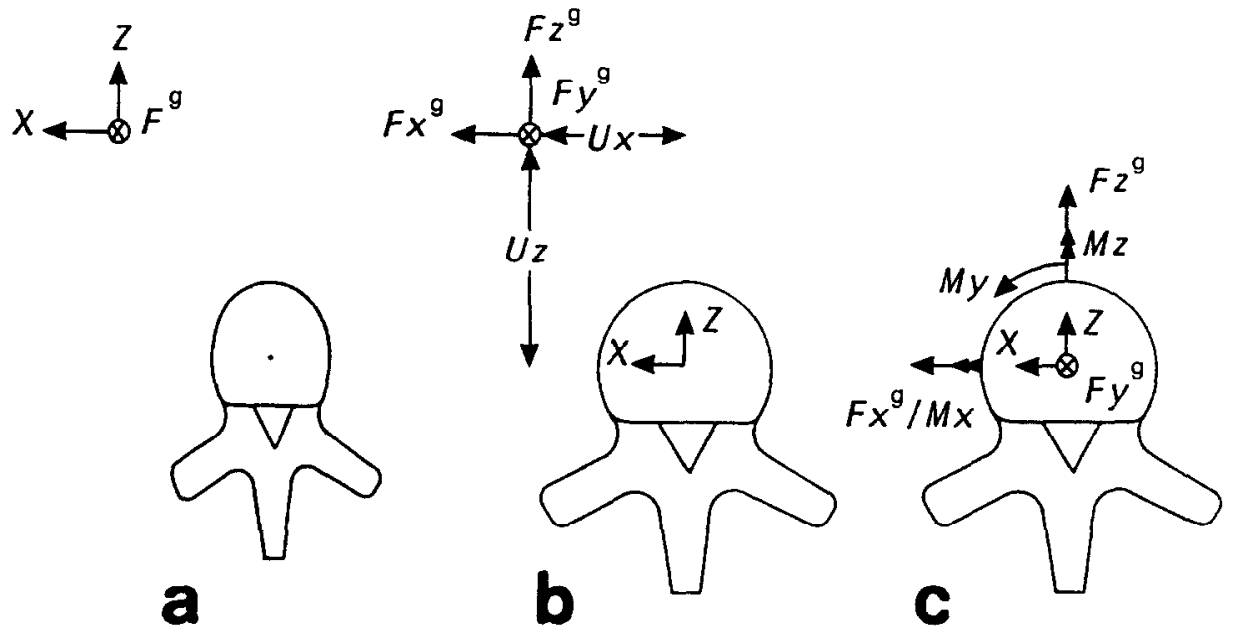

Figure 3. Influence of gravitation force on a motion segment. a, gravitation force in the global coordinate system; b, gravitation force in the local coordinate system; c, the resulting moments in the local coordinate system.

The force $F^{\mathrm{g}}(Y)$ is the gravitation force of the part of the body which lies above the spinal level $(Y)$. This gravitation force is modelled by a linear function with the value $200 \mathrm{~N}$ at level $T_{1}$ and $400 \mathrm{~N}$ at level $L_{5}$.

The line of action of the gravitation force is modelled by a straight line. This is a simplification of the reality, but it will hardly influence the qualitative results, which are the purpose of this analysis.

$F^{\mathrm{B}}(Y)=400-(0.4 \times Y)$

The function $U X(Y)$ represents the distance in the $X$ direction between the axis of axial rotation of the motion segment and the line of action of the gravitation force (see Figure 3). The function $U Z(Y)$ represents the distance in the $Z$ direction between the axis of rotation of the motion segment and the line of action of the gravitation force. These distances $U X$ and $U Z$ are a function of the level $Y$ (Figure $4 a$ and $b$ ). Figure 4 shows a distance function $U X(Y)$, from level $\mathrm{L}_{5}$ to $\mathrm{T}_{1}$, each step representing one vertebra. This distance to the $\mathrm{C}$-scoliosis is modelled by a cosine function and a constant:

$$
\begin{aligned}
U X(Y) & =A \times\left[-\cos \left(Y \times \frac{2 \pi}{L}\right)+0.6\right] \\
A & =35 \mathrm{~mm} \\
L & =500 \mathrm{~mm}
\end{aligned}
$$

The function $U Z(Y)$ consists of two parts.

The first, $U Z_{1}(Y)$, describes the sagittal curvature of the spine with a thoracic kyphosis and a lumbar lordosis (Figure 4b). In this figure the line $U Z_{1}(Y)=0$, represents the line of action of the gravitation force. The distance function $U Z_{1}(Y)$ is modelled by a third-degree polynomial function, which is an approximation of the curve of a scoliotic spine as described previously by Snijders ${ }^{5}$. In this distance function the vertebrae $T_{1}, T_{12}$ and $S_{1}$ lay on one line.

The line of action of the gravitation force is positioned $40 \mathrm{~mm}$ ventrally of $S_{1}$.

The second part consists of a translation of $40 \mathrm{~mm}$ below level $T_{11}$, caused by a shift of the axis of rotation ${ }^{2}$.

$$
\begin{aligned}
U Z_{1}(Y)= & 40-\left(6.25 \times 10^{-6} \times Y^{3}-4.375 \times 10^{-3}\right. \\
& \left.\times Y^{2}+6.25 \times 10^{-1} \times Y\right) \\
U Z(Y)= & U Z_{1}(Y)+40 \times \varepsilon(180-Y)
\end{aligned}
$$

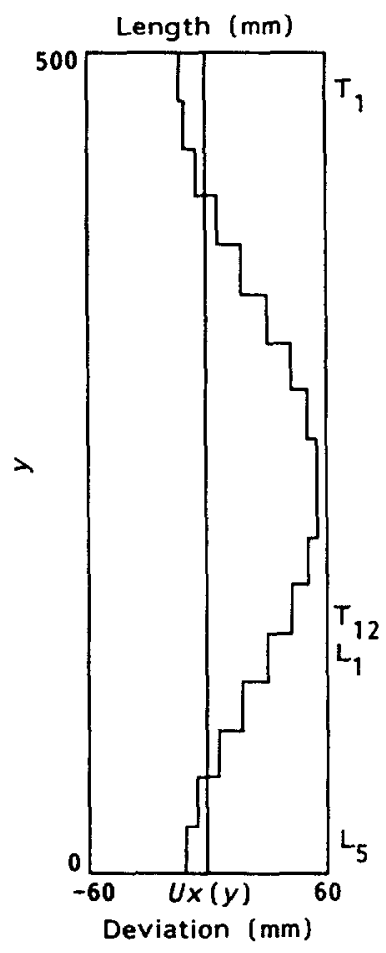

a

Figure 4. a, $U X(Y)$ is the dorsal distance of the line of action of the gravitation force to the scoliotic spine and b, $U Z(Y)$ is the sagittal distance.

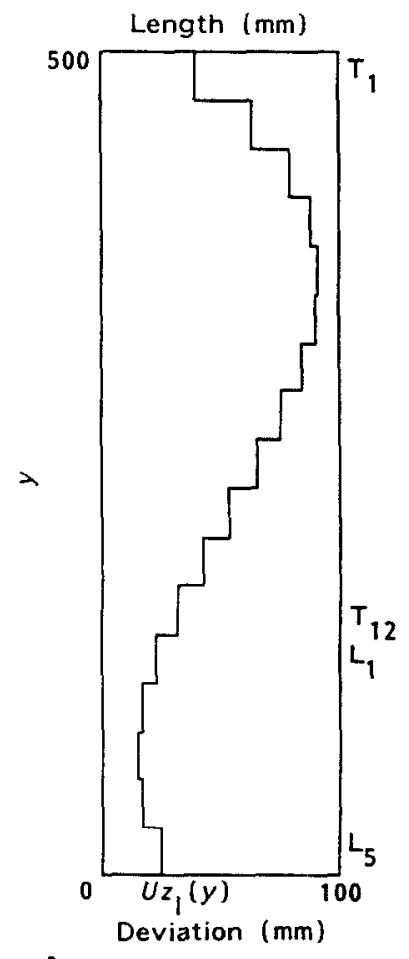

b 
From these distance functions $U X(Y)$ and $U Z_{1}(Y)$ the lateral flexion angle $\Phi_{z}(y)$ and the flexion/extension angle $\Phi_{x}(y)$ are derived. The Cobb angle for this configuration, $\Phi Z_{\max }-\Phi Z_{\min }$, is approximately 0.9 radians.

$$
\begin{aligned}
\Phi_{z}(y) & =\arctan \left(\frac{\mathrm{d}}{\mathrm{d} y} U x(y)\right) \\
& =\arctan \left(\frac{\mathrm{d}}{\cos \Phi x \cos \Phi z \mathrm{~d} Y} U X(Y) \cos \Phi_{z}\right) \\
& =\arctan \left(\frac{\mathrm{d}}{\mathrm{d} Y} U X(Y) \frac{1}{\cos \Phi x(y)}\right) \\
\Phi_{x}(y) & =\arctan \left(\frac{\mathrm{d}}{\mathrm{d} Y} U Z_{1}(Y) \frac{1}{\cos \Phi z(y)}\right)
\end{aligned}
$$

\section{Influence of correction systems on the scoliotic spine}

The influence of the Harrington and the Luque systems on the lateral deviaton and the axial rotation has been investigated.

The Harrington system uses longitudinal forces to correct the spine (Figure 5). In this model it is assumed that there are no correction forces in the local $z$ direction. From Figure 5 the local correction moments can be derived with the help of equations (1) and (2).

$$
\begin{aligned}
M y^{\mathrm{h}}(y) & =-F x^{\mathrm{h}}(y) \times U z^{\mathrm{h}}(y) \\
& =F^{\mathrm{h}} \sin \Phi_{z}(y) \times\left(\frac{U Z^{\mathrm{h}}(Y)}{\cos \Phi X(Y)}\right) \\
M z^{\mathrm{h}}(y) & =F y^{\mathrm{h}}(y) \times U x^{\mathrm{h}}(y) \\
& =F^{\mathrm{h}} \cos \Phi_{z}(y) \times\left(\frac{U X^{\mathrm{h}}(Y)}{\cos \Phi Z(Y)}\right) \\
& =F^{\mathrm{h}} \times U X^{\mathrm{h}}(Y)
\end{aligned}
$$

The force $F^{\mathrm{h}}$, the Harrington correction force, is modelled by a constant force of $300 \mathrm{~N}$.

In this case the upper hook is positioned at level $T_{6}$ and the lower hook at $\mathrm{L}_{1}$. The distance $U Z^{\mathrm{h}}(Y)$ is modelled by a constant between $T_{6}$ and $T_{11}$. Because of the shift of axes of axial rotation $U Z^{\mathrm{h}}(Y)$ has a negligible value under $T_{11}$, which is represented by the first step function. The second step function defines the position of the upper fixation level of the correction system:

$U Z^{\mathrm{h}}(Y)=40 \times \epsilon(Y-180) \times \epsilon(380-Y)$

In the model it is assumed that the deviation of the spine is a half of the original deviation when the load is fully applied'. Therefore, the distance function $U X^{\mathrm{h}}(Y)$ is defined as: half the value of $U X(Y)$, reduced by $5 \mathrm{~mm}$ to correct the distance to the fixation points. The two step functions represent the lower and
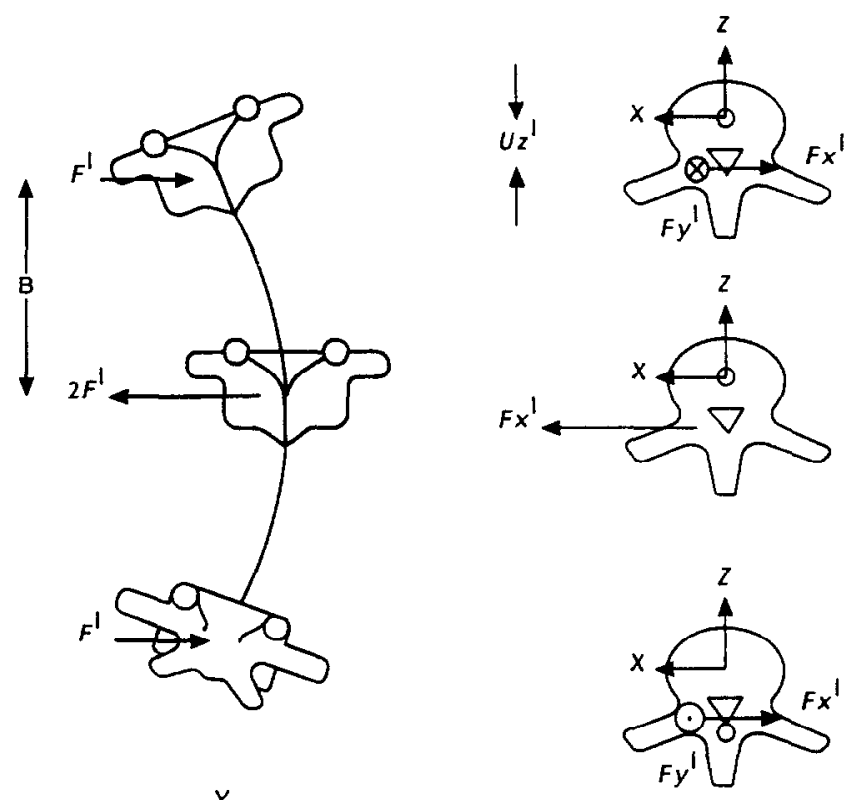

a $\quad 4$

b

Figure 5. Reproduction of the Harrington correction forces showing rotational centre, $O$. a, dorsal view of the end vertebra; $b$, cranial view of the end vertebra.

the upper fixation level of the correction system:

$U X^{\mathrm{h}}(Y)=\left[\left(\frac{U X(Y)}{2}\right)-5\right] \times \epsilon(Y-120) \times \epsilon(380-Y)$

The Luque system uses transverse forces to correct the spine (Figure 6). From Figure 6 the local moments can be derived:

$$
\begin{aligned}
M y^{1}(y) & =F x^{1}(y) \times U z^{1}(y) \\
& =F^{1} \cos \Phi x(y) \times\left(\frac{U Z^{1}(Y)}{\cos \Phi X(Y)}\right) \\
& =F^{1} \times U Z^{1}(Y)
\end{aligned}
$$
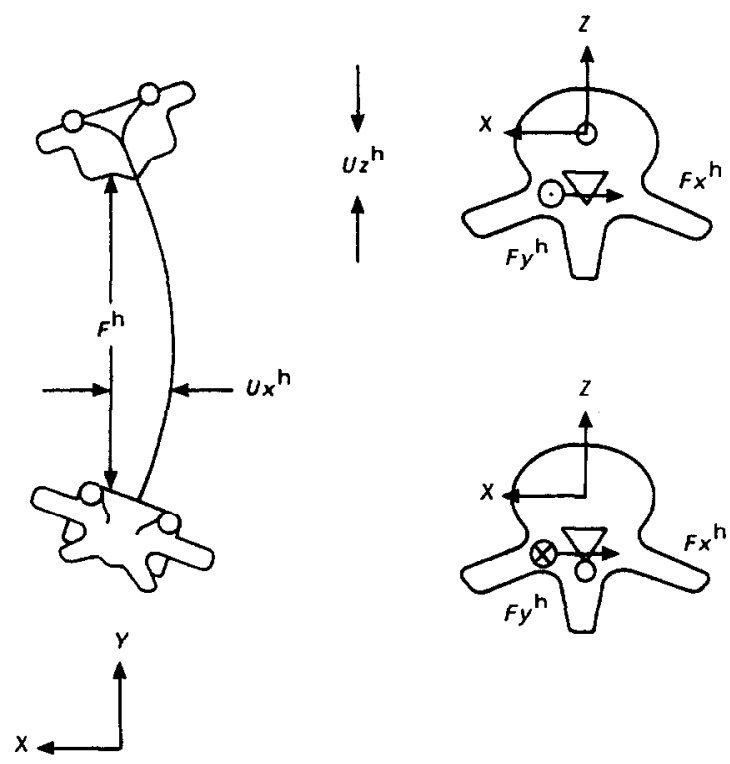

Figure 6. A reproduction of the Luque correction forces. 


$$
M z^{1}(y)=F^{1} \times B(Y)
$$

The Luque system is modelled by a three-point loading at the levels $\mathrm{T}_{6}, \mathrm{~T}_{9}$ and $\mathrm{L}_{1}$. The function $B(Y)$ describes the distance of a spinal level $(Y)$ to the outer fixation points at $T_{6}$ and $L_{1}$ :

$$
\begin{aligned}
B(Y)= & |Y-380| \times \epsilon(Y-250) \times \epsilon(380-Y) \\
& +|Y-120| \times \epsilon(Y-120) \times \epsilon(250-Y)
\end{aligned}
$$

The value of the Luque force $F^{\prime}$, is calculated so that the maximum lateral flexion moments for the Luque and the Harrington systems have the same value.

To describe the form of this corrected scoliotic spine the same functions are used as for the Harrington system.

\section{Results}

When the equations (4-9) are substituted in equations (2) and (3) the following moment diagrams, caused by the gravitation force, can be calculated (Figure 7a and b).

The axial rotation moment $M y$ is negative from $\mathrm{L}_{5}$ up to $\mathrm{T}_{12}$, with a maximum at $\mathrm{L}_{3}$ and $\mathrm{L}_{2}$. This causes a rotation of the spinous processes towards the concave side of the scoliotic curve, which has its apex between $T_{11}$ and $T_{10}$. From $T_{11}$ up to $T_{1}$ the axial rotation moment is positive, with a maximum at $T_{7}$. This causes a rotation back to the unrotated position. Therefore the moment diagram for the axial rotation is consistent with the axial rotation in the scoliotic spine, as described in literature ${ }^{1,3,6-8}$. For instance, Stokes ${ }^{7}$ states that the direction of relative axial rotation
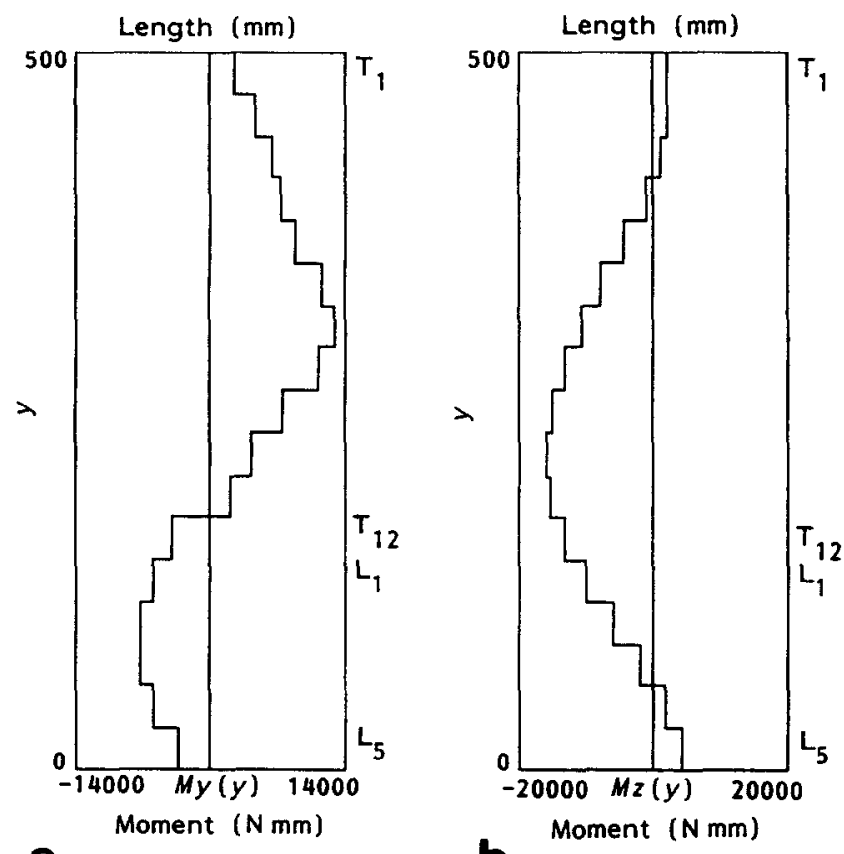

a

b

Figure 7. a, Local axial rotation moment and b. local lateral flexion moment.
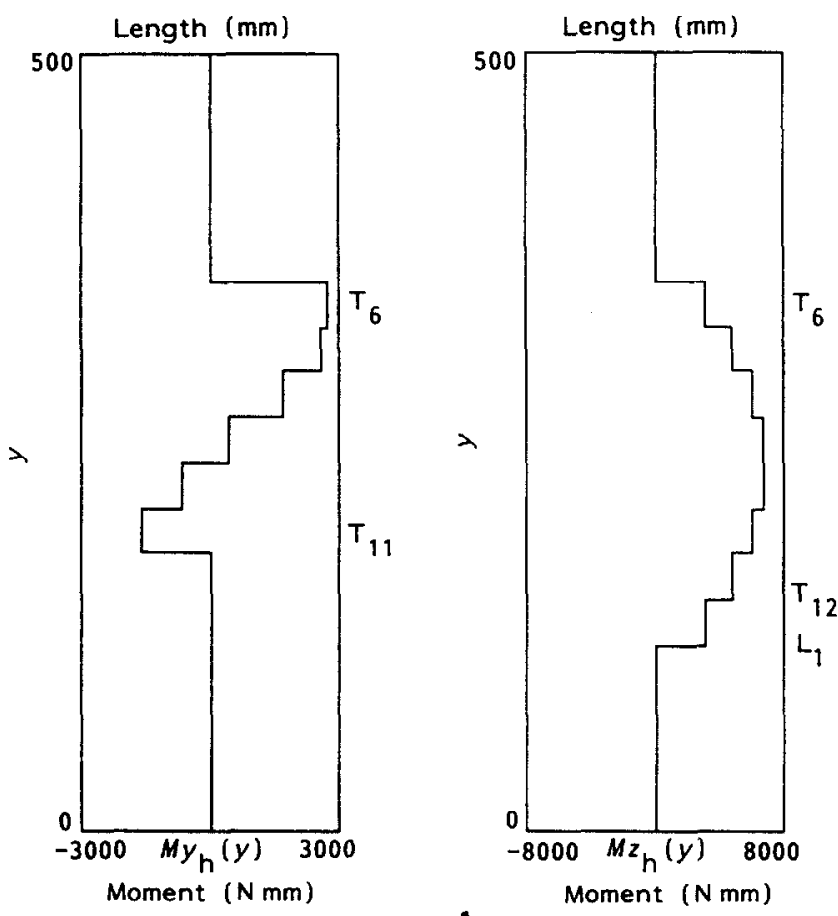

a

b

Figure 8. a, Local axial rotation moment and b, local lateral flexion moment, caused by a Harrington system, as a function of the level $y$.

between vertebrae is in an opposite sense above and below the curve apex'. And according to Veldhuizen ${ }^{3}$ 'in structural scoliosis, rotation of the vertebrae is towards the concave side of the curve'.

The lateral flexion moment, as shown in Figure 7a, is negative in almost the total curve. This means that the lateral flexion moment has a tendency to increase the existing lateral deviation.

The moments necessary for the correction of the scoliotic spine should rotate the vertebra in the opposite direction to the rotations caused by the gravitation force. It is therefore plausible that the correction moments have to be the opposite to the gravitation moments.

When the equations (12) and (13) are substituted in (10) and (11) the moment diagrams $M y^{\mathrm{h}}$ and $M z^{\mathrm{h}}$ for the Harrington correction can be calculated (Figure 8a and b). These moment diagrams $M y^{\mathrm{h}}$ and $M z^{\mathrm{h}}$ should, as stated before, be the opposite of $M y$ and $M z$ (Figures $7 \mathrm{a}$ and $\mathrm{b}$ ). This is correct for the lateral flexion moment $M z^{\mathrm{h}}$ as shown in Figure $7 \mathrm{~b}$ but the axial rotation moment $M y^{\mathrm{h}}$ has the same sign as $M y$ at level $T_{8}-T_{6}$ and will therefore increase the rotation in this part of the spine. At level $\mathrm{T}_{11}-\mathrm{T}_{10}$ the axial rotation moment $M y^{\mathrm{h}}$ has the opposite sign of $M y$ and will therefore decrease the axial rotation.

Next the correction moments for the Luque system are calculated, by substituting equations (12), (13) and (16) in equations (14) and (15) (Figure 9a and b). Here too, the moment diagram for the lateral flexion $M z^{1}$ is the opposite of $\mathrm{Mz}$ which is the correct shape. But the moment diagram for axial rotation $M z^{1}$ is totally 


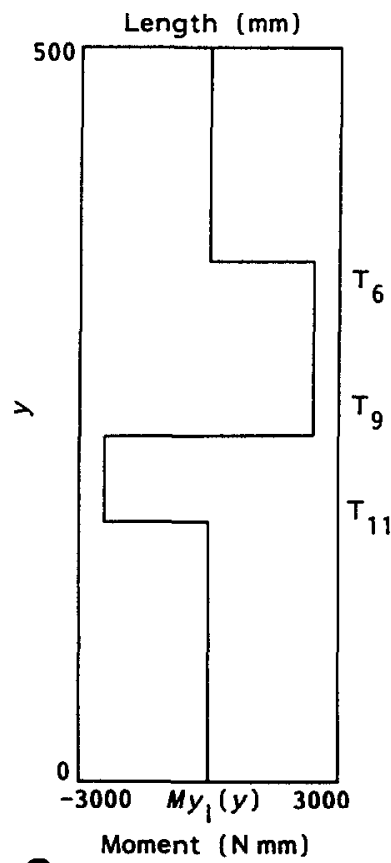

a

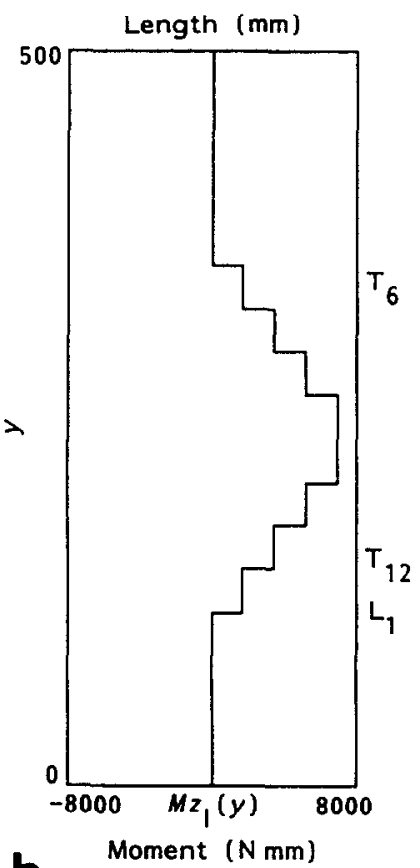

b

Figure 9. a, Local axial rotation moment and b, local lateral flexion moment, caused by a Luque system, as a function of the level y.

different and will enlarge the rotation, mainly between $T_{9}$ and $T_{6}$.

\section{Discussion}

In the model, as presented before, the influence of an eccentrical gravitation force on a spine with a lateral deviation has been investigated. The influence of other forces, as muscle or ligament forces, have not been included in the model. These forces will try to compensate for the moments caused by the gravitational force. But if they, for some reason, are not capable of doing so, the resulting moments will tend to worsen the slightest deviation. This means an increasing lateral deviation and an axial rotation towards the concave side of the curve. This explanation is the opposite to that given by White ${ }^{8}$, who states that an imbalance causes axial rotation which in its turn causes the lateral deviation. It is the view of Stokes ${ }^{7}$ that there cannot be a simple coupling between lateral bending and axial rotation. The authors agree with this statement because the sagittal curvature of the spine and the position of the axis of axial rotation have to be considered for the explanation of the coupling between lateral bending and axial rotation.

In the model, the spine is modelled in the form of a $\mathrm{C}$-scoliosis, but the results of another model with an

S-scoliosis were consistent with the results as presented before.

For both the investigated correction systems, Harrington and Luque, the moments for the correction of the axial rotation are very unfavourable. The maximum values for these moments are almost the same for the given spinal form. But when a less severe scoliosis is investigated, the influence of the Luque system on the axial rotation is worse than the Harrington system.

\section{Conclusions}

1. The axial rotation in the scoliotic spine can be explained by the influence of the eccentrical gravitation force.

2. The Harrington and Luque systems tend to increase the axial rotation in the scoliotic spine.

3. The Harrington and Luque systems produce favourable moment diagrams for correction of the lateral flexion.

\section{Acknowledgement}

This research was supported by the Technology Foundation (STW).

\section{References}

1 Scholten P. Idiopathic scoliosis, some fundamental aspects of the mechanical behaviour of the human spine [PhD Thesis]. Amsterdam, 1986

2 Singer KP, Breidahl PD, Day RE. Posterior element variation at the thoracolumbar transition. Clin Biomech $1989 ; 4: 80-6$

3 Veldhuizen AG. Idiopathic scoliosis [PhD Thesis]. Groningen, 1985

4 Haha T, Feliny W, O'Brien M. The instantaneous axis of rotation as a function of the three columns of the spine. 24th Annu Meeting Scoliosis Res Society, Amsterdam, 1989, pp 316-7

5 Snijders CJ. On the form of the human thoracolumbar spine and some aspects of its mechanical behaviour [PhD Thesis]. Eindoven: Technical University of Eindoven, 1970

6 Subbaraj K, Ghista DN, Viviani GR. Presurgical finite element simulation of scoliosis correction. J Biomed Eng $1989 ; 11: 9-18$

7 Stokes IAF. Axial rotation component of thoracic scoliosis. J Orthop Res 1989; 7: 702-8

8 White AA, Panjabi MM. Clinical Biomechanics of the Spine. Philadelphia, Toronto: JB Lippincott Co., 1978, Chapter 3

9 Harrington. Treatment of scoliosis correction and internal fixation by spine instrumentation. J Bone Joint Surg 1962; 52A: 498-506

10 Luque ER, Ramirez-Wiella G. Segmental spinal instrumentation in the treatment of the spine. Spine 1982; 7: $312-7$ 